\title{
Video Technology as an Aid for Stimulation: An Insight
}

\author{
Swetha Grandhi ${ }^{1}$, Monika Suresh ${ }^{2}$, Akhila S. Rajan ${ }^{3}$ \\ ${ }^{1}$ Lecturer, Department of Speech Language Studies, Dr. S.R. Chandrasekhar Institute of Speech and Hearing, Lingarajapuram, Hennur Main \\ road, Bangalore-560084, Karnataka, India \\ ${ }^{2}$ Graduate Clinician, Department of Speech Language Studies, Dr. S.R. Chandrasekhar Institute of Speech and Hearing, Lingarajapuram, \\ Hennur Main road, Bangalore-560084, Karnataka, India \\ ${ }^{3}$ Graduate Clinician, Department of Speech Language Studies, Dr. S.R. Chandrasekhar Institute of Speech and Hearing, Lingarajapuram,
} Hennur Main road, Bangalore-560084, Karnataka, India

\begin{abstract}
Communication disorder is impairment in the ability to receive, send, process, and comprehend verbal, nonverbal and graphic symbol systems. Counselling is an integral clinical responsibility for families withchildren and adult who have acquired such disorders. Technology has the potential to benefit individuals with communication disorder throughout their lifespan. One of the innovative ways for intensive speech and language stimulation is use of video technology.Hence there is a need to understand the technological advancement in achieving the success for persons with disability. Study design guided the parents to use the video technology as a stimulatory aid. Results revealed that there is a progress in the targets through video modelling, enhanced the knowledge of the parents about the method of teaching the child.Thus it can be concluded that this research will help the speech language pathologist, to enhance knowledge about the technological advancements which can serve the persons with disability.
\end{abstract}

Keywords: Communication, Videotechnology, Stimulatory aid.

\section{Introduction}

American Speech and Hearing Association (1982) defined Communication disorder as an impairment in the ability to receive, send, process, and comprehend verbal, nonverbal and graphic symbol systems.As a Speech language pathologist thorough knowledge in the identification of the presence or absence of the problem followed by a detailed assessment to intervene the problem should be carried out with adequate counselling sessions (American Speech and Hearing Association, 2003).

Counselling is an integral clinical responsibility for families and children with speech, language, and hearing disorders, as well as for adults who have acquired such disorders (American Speech and Hearing Association, 2001). As a diagnostician, our clinical training and professional skills should focus on counselling the specific needs of parents, understandingchild's problem and importance of intensive speech and language stimulation.

There are various methods to stimulate the child at home. One of the innovative ways for intensive speech and language stimulation is use of video technology. With the advent of cheaper, smaller, and more portable technological devices, more and more families and professionals are turning to electronics as a support for parents of children with communication disorders. Technology has the potential tobenefit individuals with communication disorder throughout their lifespan. There has been an increased interest in the use of technology for these children over the past few years, especially in the application of computers, smartphones, iPads, iPods, and other high tech opportunities to teach communication skills and facilitate the use of a wide variety of other skills.
Video modelling and self-modelling have gained their importance widely compared to that of other modelling in the recent decades. Videomodelling is a form of observational learning in which desired behaviours are learned by watching a video demonstration and then imitating of the behaviour of the model. According to Neumann (2004) video modelling is the procedure of videotaping targeted behaviours in order to expand the learner's capability to memorize, imitate, and generalize or adapt targeted behaviours.

Video self-modelling is an intervention procedure similar to video modelling. Video camera records the entire output delivered by the child and edit to include only the positive target behaviour. The subject, as the self-model, repeatedly views the video clip for two to four minute in order to learn the target behaviours (Dowrick, 1999).

Dowrick \& Bandura (1991) stated that Video self-modelling (VSM) is a technique that allows Video feed forward, positive self-review and increases self-efficiency. In video self-modelling (VSM), individuals observe themselves performing behaviour successfully on video, and then imitate the targeted behaviour. Video modelling has been used to teach many skills, including social and communicationskills.Video modelling and self-modelling assists as an aid in using the technology for persons with disability (PWD).

\section{Literature Survey}

Bellini and Akullian (2007) considered videomodelling as an evidence-based practice.Christos et.al (2004) grounded their research to examine the effects of a video modelling intervention on social initiation and play behaviours, in 3 children with autism spectrum disorders. Each child watched 


\section{International Journal of Science and Research (IJSR) \\ ISSN (Online): 2319-7064}

Index Copernicus Value (2013): 6.14 | Impact Factor (2014): 5.611

a videotape showing a typically developing peer, and the experimenter engaged in a simple social interactive play using one toy. For all children, social initiation and reciprocal play skills were enhanced, and these effects were maintained at 1- and 3- month follow-up periods.

Genevieve \&Rutherford (2014) studied the effectiveness of video self-modelling (VSM).Researchers suggested that VSM is effective across a range of ages, behaviours andvideo self-modelling (VSM) helps in teaching variety of skills, including math, life skills, social behavioursand language.It also helps in treating various disorders like depression, stuttering, attention and aggressive behaviours. Thus it can be concluded that these behavioural improvements facilitate and enhance their involvement in the community. With increasing support on the usefulness of this intervention, video self-modelling has the potential to be used as a tool to effectively help more individuals in the near future.

Burton et.al (2013) focused their research in understanding the use of video self-modelling on an iPad to teach functional math skills to four adolescents with intellectual disability.Video self-modelling (VSM) was implemented twice daily, 4 days a week using a flip video camcorder, Windows Live Movie Maker, and an iPad. Five different videos were created for each participant. Student one had a $74 \%$ increase in correct responses, student two had an $86 \%$ increase in correct responses, student three had a $98.5 \%$ increase in correct responses, and student four had a $84.5 \%$ increase in correct responses using Video self-modelling. Results revealed significant improvement in student performance. Thus it can be concluded that video selfmodelling may be considered as an effective technologybased intervention for students with intellectual disability in the classroom.

Gelbar (2011)suggested that Video self-modelling (VSM) is successful in increasing language and communication skills, social skills, and task instruction. An effect of a Video Model to teach three Students with Moderate Intellectual Disability to Understand Key Features of an iPhone was studied by Walser et.al (2012).A multiple probe design across three behaviours, replicated across three participants, was used to evaluate the effects of video modelling on participants' capacities to (a) take a photograph of a person, (b) look at photographs by starting a slideshow, and (c) access and view a video.Results of the present study indicate that video modelling was effective in teaching target behaviours in a near-errorless fashion.

\section{Need for the Study}

In the past few years, the emphasis and importance of science, technology, engineering, and mathematics education for today's youth has exploded. Unfortunately, there is little research on implementing the technological innovations for rehabilitation services of parents of children with disability as a stimulatory aid.Hence there is a need to understand the technological advancement in achieving the success for persons with disability.

\section{Aim of the Study}

The aim of the study is to guide the parents to understand the video technology and to use it as a stimulatory aid for a child with disability.

\section{Method}

A 12 year old male child with a history of delayed birth cry, febrile seizure and delayed developmental mile stones came to the institute with a concern of not speaking age appropriately. Detailed speech and language assessment carried out using formal tests and the results revealed inadequate speech and language skills secondary to cognitive deficits. Psychological assessment reveals moderate deficits (IQ-43).After obtaining a provisional diagnosis parents were counselled on the importance of speech and language therapy and the role of parents in intensive speech and language stimulation at home.

Speech and language therapy focused on improving the prelinguistic skills, increasing the mean length of utterance (MLU)and also improving the correct production of sounds like /s/ and /th/.After 1 month of speech therapy sessions, a pre therapy assessment questionnaire is given to the parents in order to evaluate the intensive speech and language stimulation at home. The questionnaire developed by speech language pathologist addressing various aspects such as Importance of speech therapy, rationale in using various speech stimulation technique, modelling the child at home, adequate duration spent with the child for practise, need of assistance in practising, feedbacks given by the parent on errors exhibited by the child, generalizing the technique while speaking, self-initiation of the child to use the technique and time spent to model the child under various situation.

All the targets were planned to address through video technology. In order to implement the advantage of using the technology speech language pathologist should really put an effort in obtaining perfect videoswith less distraction that address the targets of the client., the targeted goals are reducing rate of speech, increasing the mean length of utterance, eye contact, Attention span and concentration. The equipment used to record the session was canon digital camera.Cue cards, visual cues were used to elicit the target behaviours. Video recording was done for 3 consecutive therapy sessions (i.e. $45 \mathrm{~min}$ per session).Informed consent was taken from the parent prior to the recording of all the sessionsand only the positive responses of the client were edited using Windows movie maker. All the video recorded sessions were analysed by following the steps provided by National Profession Developmental Centre on Autism Spectrum Disorder (2008).

The edited responses for video self-modelling were divided into 4 categories such as, client conversation, storytelling, monologue and general behaviours. Video modelling recordings done by the clinicians includedcorrect production of $/ \mathrm{s} /$ and $/$ th/ sounds, correct conversation model, modelling correct and incorrect behaviours, steps to be remembered while speaking.Videos given to the parent includes videos of 


\section{International Journal of Science and Research (IJSR) \\ ISSN (Online): 2319-7064 \\ Index Copernicus Value (2013): 6.14 | Impact Factor (2014): 5.611}

self-modelling, video modelling and also tips for parents focusing on the targets.

Parents were explained about the importance of videos and were asked to view these videos along with the client on a regular basis. Parents were also counselled to use some instructional delivery to the client like "It's time to watch your movie".All the videos were observed by parents for 1 month and then a post therapy questionnaire was given to the parent addressing, various aspects such as Importance of using video at home to achieve the targets, Rationale in using the videos,time spent at home for practising with videos,assistance from parents to practise the technique using videos, modelling the child using videos, Feedbacks given for exhibiting errors, Maintenance of eye contact, Generalizing positive and negative behaviours by the child after using video, Role of videos in helping both parent and child.

For each question the 5-point Likert rating scale (Always $100 \%$, Frequently $75 \%$, Occasionally $50 \%$, Seldom $25 \%$, Never $0 \%$ ) scoring was represented. Pre and post rating was marked by the parents accordingly.

\section{Results}

The current study aimed to guide the parents to understand the video technology and to use it as a stimulatory aid for a child with disability.

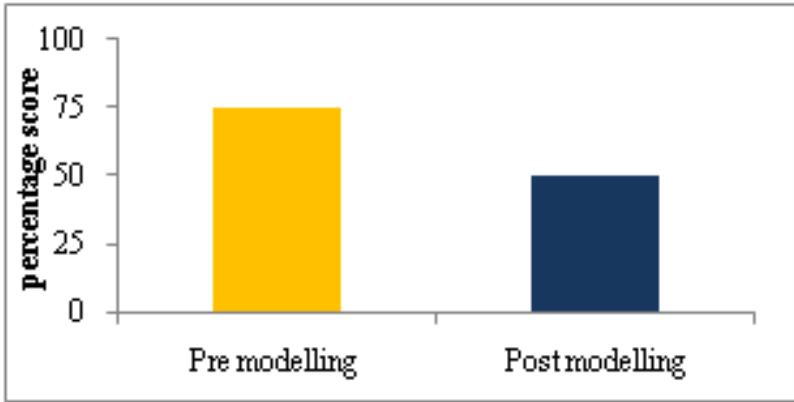

Figure 1: Representation of Parental modelling

From figure 1 we understand that before the presentation of video, $75 \%$ of time the parent had to model the child.But after receiving the video the parent's role of modelling reduced to $50 \%$ as the video helped the child to understand the target.

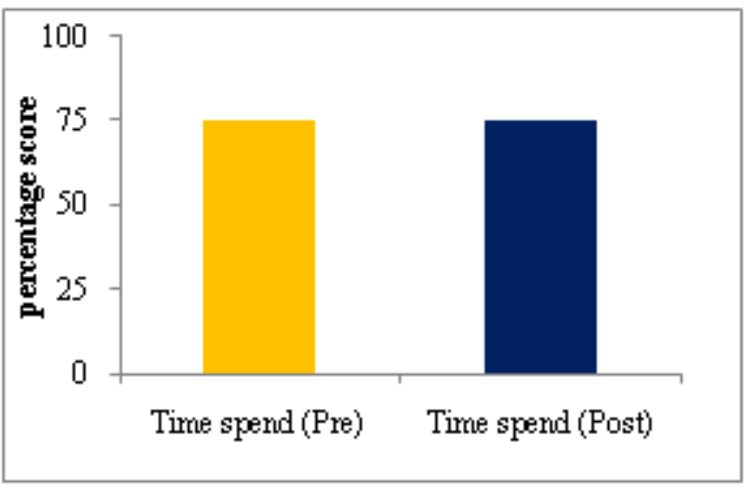

Figure 2: Amount of time spend by Parents
From the above figure one can infer that the amount of time spend by the parents with the child for stimulation was observed to be the same (i.e. $75 \%$ ).

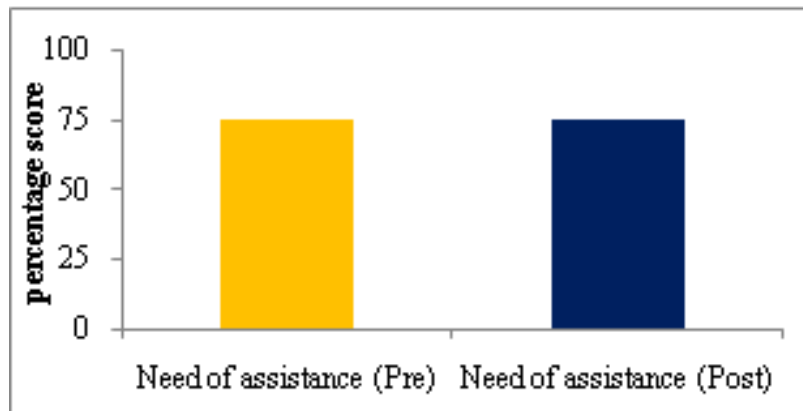

Figure 3: Assistance to practise the technique at home.

From figure 3 it is evident that the child requires (75\%) of assistance from the parents to practise the technique before and after introducing to give the video models of the child to parents.

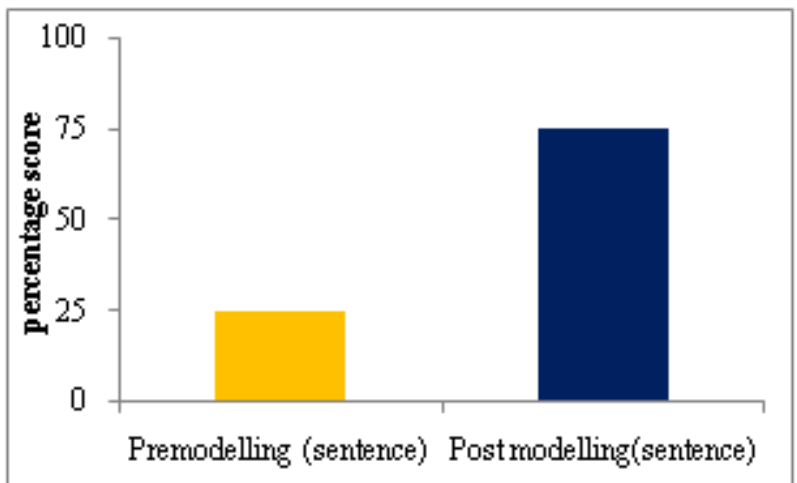

Figure 4: Pre and Post modelling of speaking in sentences

Figure 4 depicts that beforereceiving the video parents model the child to use the technique only $25 \%$ of the time. But after receiving the video parents were modelling $75 \%$ of time and they understood the importance of modelling the child to speak in sentences.

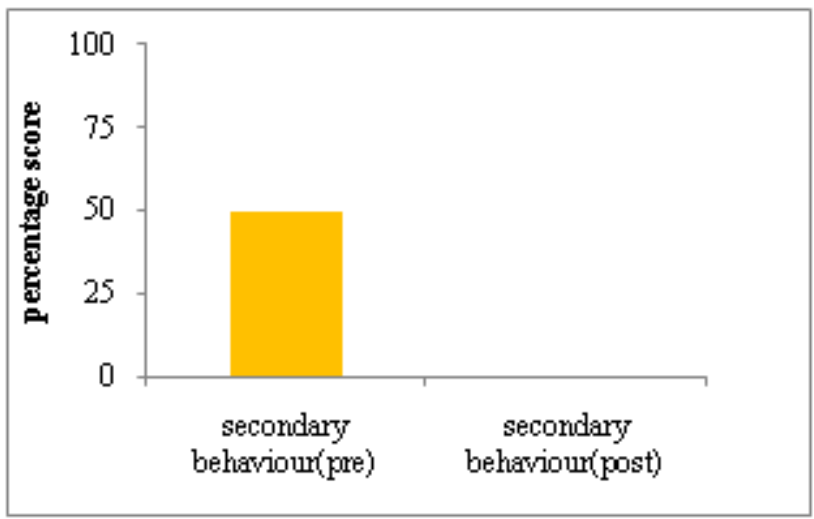

Figure 5: Modelling the child on secondary behaviours

From figure 5 it is clear that parents model the child 50\%of time not to exhibit secondary behaviours but after receiving the video the parental modelling was not necessary and the child understood not to exhibit the secondary behaviours during speaking. 


\section{International Journal of Science and Research (IJSR) \\ ISSN (Online): 2319-7064}

Index Copernicus Value (2013): 6.14 | Impact Factor (2014): 5.611

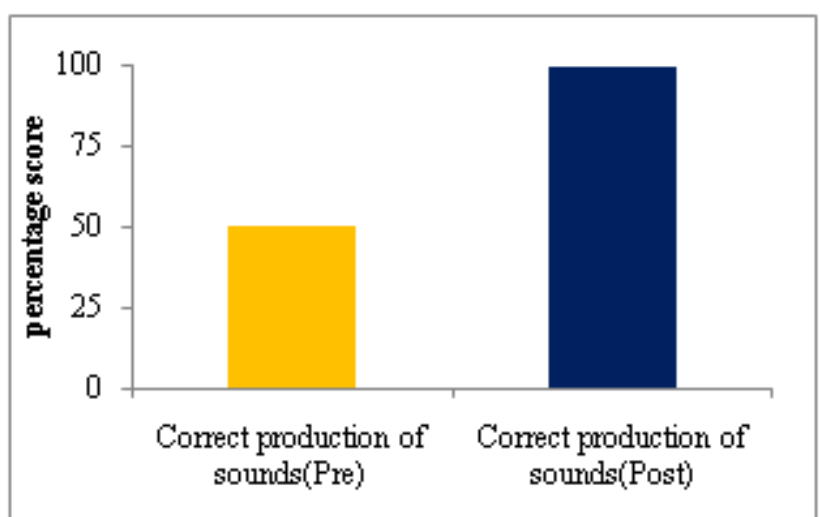

Figure 6: Representation of correct production of sounds $(/ \mathrm{S} / \& / \mathrm{th} /)$

Figure 6 depicts that before video modelling the child was able to produce the sound $/ \mathrm{s} /$ and $/ \mathrm{th} /$ only $50 \%$ of time. Video modelling helped the child understand the correct place and manner of articulation for the sound $/ \mathrm{s} /$ and $/ \mathrm{th} /$.

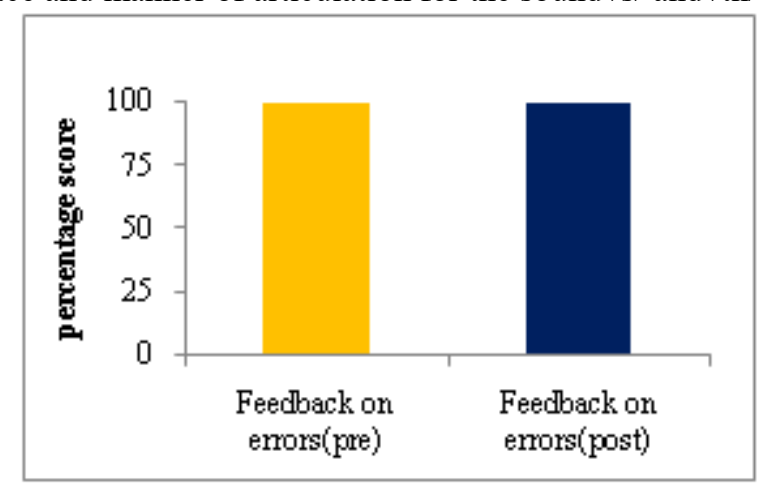

Figure 7: Feedback on the errors (Pre\&Post)

From figure 7 one can infer that parental feedback was $100 \%$ both before and after the video.

\section{Conclusion}

Video modeling is a form of video based intervention (VBI) which includes visual and auditory modalities to elicit the desired behavior. In the present study information based on parentalquestionnaire reveals that there is a progress in the targets through video modeling, enhanced the knowledge of the parents about the method of teaching the child. Thus as a speech language pathologist, we should always enhance our knowledge about the technological advancements which can serve the persons with disability.

\section{References}

[1] Hitchcock, C., Dowrick, P. W., \&Prater, M. A. (2003). Video self-modeling interventions in school-based settings: A review. Remedial and Special Education, 24, $36-46$.

[2] Sigafooso'Reily,De la cruz(2007) Using technologies to develop capabilities with people living with intellectual disabilities.

[3] Dowrick, P.W. (1991). Practical guide to using video in the behavioral sciences. New York: Wiley Interscience.

[4] Dowrick, P.W. (1999). A review of self-modeling and related interventions. Applied and Preventive Psychology, 8, 23-39.
[5] Bandura, A. (1982). Self-efficacy mechanisms in human agency. American Psychologist, 37 , 122- 147.

[6] Nikopoulos, C. K., \& Keenan, M. (2004). Effects of video modeling on social initiations by children with autism. Journal of Applied Behavior Analysis, 37(1), 93-96.

[7] Genevieve Hin,HaTsui and Rutherford (2014).Video Self-Modeling Is an Effective Intervention for an Adult with Autism

[8] Bellini, S. \& Akullian J. (2007), A meta-analysis of video modelling and video self-modeling interventions for children and adolescents with ASD. Exceptional Childern, 73, 261-28.

[9] Buggey, T. (2007). Video self-modeling applications at school and at home. Journal of Positive Behavior Interventions, 9, 151-158.

[10] Cami Elizabeth Burton(2013). Video Self-Modeling on an iPad to Teach Functional Math Skills to Adolescents with Autism and Intellectual Disability.

[11] Nicholas. W.Gelbar,Candace Anderson, Scott McCarthy,TomBuggey (2011).Video self-modeling as an intervention strategy for individuals with autism spectrum disorders.

[12] Kathryn Walser, Kevin Ayres and Erika Foote Education and Training in Autism and Developmental Disabilities Vol. 47, No. 3 (September 2012), pp. 319 331.Effects of a Video Model to Teach Students with Moderate Intellectual Disability to Use Key Features of an iPhone.

[13] National Professional Developmental Centre on Autism Spectrum Disorder (NPDC on ASD) (2008).

[14] American Speech and Hearing Association (1982).

[15] American Speech and Hearing Association (2003).

\section{Author Profile}

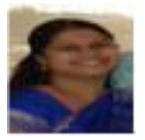

G. Swetha presently working as a lecturer in the Department of Speech and Language Studies in Dr.S.R. Chandrasekhar Institute of Speech and Hearing, Bangalore, Karnataka, India. I am an active participant in research and also guided students for the research related activities. And my core areas of interest are child language disorders, fluency and voice. I have presented and published many research articles in the National Level Journals and also in the proceedings.

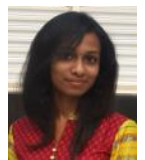

Monika Suresh, a graduate student (Bachelors in Speech Language Pathology and Audiology) from Dr.S.R Chandrasekhar Institute of Speech and Hearing Bangalore, Karnataka, India. As a student I am an active participant in research activities.

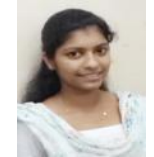

Akhila.S.Rajan, a graduate student (Bachelors in Speech Language Pathology and Audiology) from Dr.S.R Chandrasekhar Institute of Speech and Hearing Bangalore, Karnataka, India. As a student I am an active participant in research activities. 